\author{
Military Technical College \\ Kobry Elkobbah, \\ Cairo, Egypt
}

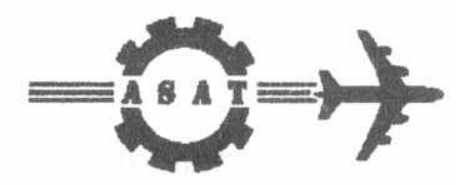

$8^{\text {th }}$ International Conference on Aerospace Sciences \& Aviation Technology

\title{
NOISE EFFECT ON GUIDANCE AND CONTROL LOOP OF GUIDED BOMBS
}

\author{
AMIR H. MAKARYUS * AHMED S. GAD**
}

\begin{abstract}
:
Smart or homing guided bombs are used to destroy difficult-to-hit targets with minimum number of strikes and high accuracy. There are two basic types of smart bombs, laser or TV guided bombs. The guided bomb is formed by adding a seeker and a control section to the traditional unguided bombs. The seeker searches for and tracks the target. It provides the line-of-sight rate to the guidance module that generates and provides guidance commands to the control fins. The autopilot of the guided bomb is mainly used to improve the accuracy, maintain a near constant steadystate aerodynamic gain, and help in steering the bomb to the target with a very high accuracy.
\end{abstract}

Undesirable behavior characteristics of the guidance and control may originate due to several sources of disturbances such as flight vibrations and oscillations due to external environmental effects and/or the airframe coupling mechanisms flutter or the propulsion system motor chamber pressure oscillations. The latter will produce phasecoherent quasi-sinusoidal vibration while the former is random and unpredicted and can only be evaluated using statistical methods of analysis.

In this paper, the external noise presented by the atmospheric turbulence during the bomb flight is investigated through computer simulation. The noise process is modeled and generated to enable evaluation of the system performance. The zeromean white Gaussian noise is generated and provided as a superimposed input with the acceleration input command of the bomb autopilot. The system performance is measured for different signal-to-noise ratios (SNR) of the input noise. The performance evaluation is made via a six-degrees-of-freedom (6-DOF) flight model of the bomb. The measured parameters are the trajectory, the final missdistance, and the autopilot acceleration response.

\footnotetext{
* Ass. Prof., Radar and Guidance Dpt., Military Technical College, Cairo, Egypt.

*AAss. Lecturer, Radar and Guidance Dpt. , Military Technical College, Cairo, Egypt.
} 


\section{INTRODUCTION:}

In the analysis and design of practical systems, the use of sinusoidal, step, or impulse functions only as system stimuli inputs is insufficient. The designer may design an autopilot that gives extremely good response to a step or a pulse input, but how will this system behave under the influence of randomly varying input signals which represents the atmospheric turbulence? If the system responds rapidly, which means a high gain. Then, in the presence of turbulence excessive acceleration loads may be imposed on the airframe. This requires the system response to be optimized to operate accurately under the most adverse conditions, that is, extremely low signal-tonoise ratio, [1].

The atmospheric turbulence forms a random input that cannot in general be reproduced analytically. True, the behavior of the autopilot under the influence of noise inputs can be simulated using a noise generator to furnish the disturbing inputs; however, it would be useful to have an arialytical means for predicting the performance of the system to these random inputs during the design phase. This will provide the control system engineer with necessary tools to predict the response of a system to random inputs, [2,3].

\section{STATISTICAL MODELING OF NOISE EFFECT}

The effect of the external noise and the internal fluctuations of the guidance loop elements on the operation of the control system poses a very complex mathematical problem owing to the specific nonlinearity of the system.

The guidance loop equation is a nonlinear, stochastic integro-differential one, which leads in the presence of noise to several difficulties. Among these is the meaning of the solution of the differential equation driven by a random signal. The determination of the steady-state flight conditions is no longer a simple evaluation of the response of the loop to the demanded kinematics conditions. An important issue is the selection of compensators in the loop to optimize its performance.

As a first approach, which is a special but an important practical one, the noise process will be considered additive, independent, and delta-correlated white Gaussian with zero mean. To model the noise and random error sources in guidance and control system, it is necessary to generate a sequence of random inputs. The random process is characterized by the standard deviation or the power spectral density function.

If the random variable $X$ is defined as the instantaneous value of the turbulence (atmospheric noise) measured, there will be a certain probability density or distribution function, $f(x)$, such that

$$
P(x<x<x+d x)=\int_{x}^{x+d x} f(\alpha) d \alpha
$$


Equation 1 states that the probability that the instantaneous value of the noise lies within the range of $x$ to $x+d x$ is equal to the probability density function integrated over the range of values, $d x$. If it is assumed that the values of the noise follows a normal or Gaussian distribution, then

$$
f(x)=\frac{1}{\sigma \sqrt{2 \pi}} e^{-(x-m)^{2} / 2 \sigma^{2}}
$$

Where $\mathrm{m}$ is the mean or the average value of $\mathrm{x}, \sigma$ is the standard deviation, and $\sigma^{2}$ is the variance. It is assumed that the mean value of the noise over a long flight path is zero, then $m=0$, and the probability density function will be symmetrical about the $x=0$ axis. The noise then is called a zero-mean white Gaussian noise. The random process is a series of probability density functions. A set of these probability density functions completely defines the statistical characteristics of the random process.

It is important to select a measure of the error to be minimized, as this determines which statistical characteristics of the input are important, [4]. The measure of the error is the mean-square error, which is defined as

$$
\overline{e^{2}}=\lim _{T \rightarrow \infty} \frac{1}{2 T} \int_{-T}^{T}\left[f_{0}(t)-f_{d}(t)\right]^{2} d t
$$

Where $f_{o}(t)$ is the actual acceleration output and $f_{d}(t)$ is the desired acceleration output. The mean-square error can be expressed in terms of certain statistical characteristics, such as the correlation function or the power spectral density.

Power spectral density (PSD) function is used to describe the distribution of noise power with frequency, in units ${ }^{2} / \mathrm{Hz}$. Thus, the PSD of angular noise is expressed in $\mathrm{rad}^{2} / \mathrm{Hz}$ and glint noise in $\mathrm{m}^{2} / \mathrm{Hz}$. White noise extends over all frequencies and is flat. Band-limited noise can be treated as a white noise signal that has been passed throughout a first-order filter with a bandwidth equivalent to a specific band limit. The PSD is mathematically defined as the Fourier transform of the autocorrelation function of the signal for which the PSD is to be determined. A PSD function can be physically realized by passing the noisy signal through a set of narrow bandpass filters, computing the square of the output from each filter, and plotting root mean-square value (RMS) of the squared output, mean power, versus the center frequency of the filter. If the signal is pure white noise, the mean power from each filter will be equal. If the signal is filtered, or band-limited, the RMS power versus frequency will look like the amplitude ratio plot of the filter transfer function. The total power in a signal is the integral of the PSD function over frequency.

The autocorrelation function is an important statistical characteristic of any signal. It is a measure of the predictability of the signal at some future time based upon the present knowledge of the signal, [5]. The autocorrelation function of the bandlimited white noise is sampled in the form of narrow pulses of duration $\Delta t$ and 
amplitude a. The autocorrelation function of this sampled noise is given by equation (4) and shown in fig. 1.

$$
\phi_{a}(\tau)=a^{2}\left(1-\frac{|\tau|}{\Delta t}\right)
$$

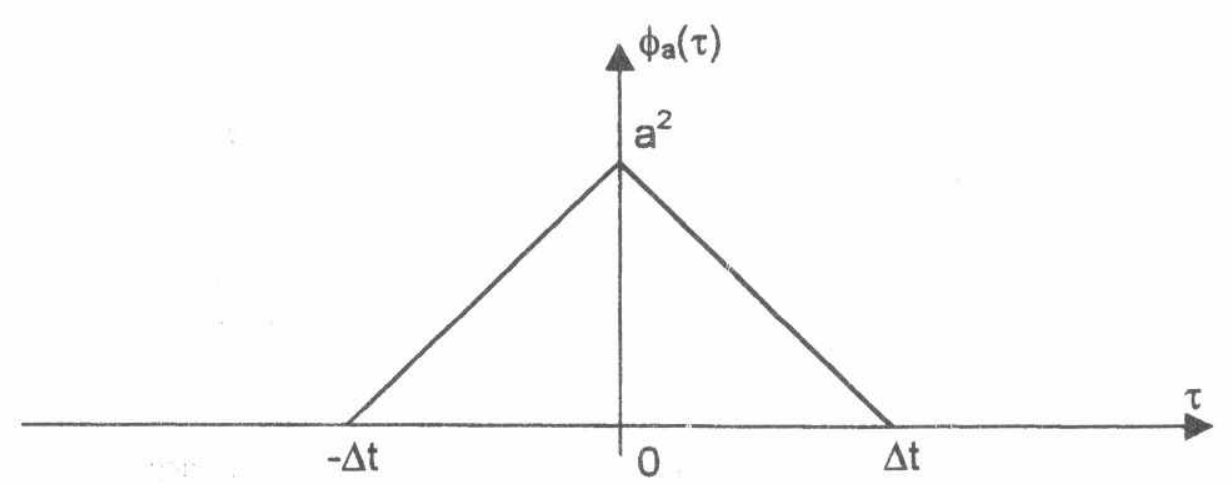

Fig. 1 Autocorrelation function of band-limited white noise

It is useful to be able to work in the frequency domain. The Fourier transform is used to change from time to frequency domain. The PSD is denoted by

$$
\Phi_{\mathrm{a}}(\omega)=\int_{-\infty}^{\infty} \phi_{\mathrm{a}}(\tau) e^{-\mathrm{j} \omega \tau} \mathrm{d} \tau
$$

But,

$$
e^{-j \omega \tau}=\cos \omega \tau-\cdot j \sin \omega \tau
$$

So,

$$
\Phi_{\mathrm{a}}(\omega)=\int_{-\infty}^{\infty} \phi_{s_{A}}(\tau)(\cos \omega \tau-j \sin \omega \tau) \mathrm{d} \tau
$$

The sine wave is an odd function and the integral from minus infinity to plus infinity is zero. So, the second term of equation (6) is omitted.

$$
\begin{aligned}
& \Phi_{\mathrm{a}}(\omega)=\int_{-\Delta t}^{\Delta t} \mathrm{a}^{2}\left(1-\frac{|\tau|}{\Delta t}\right) \cos \omega \tau d \tau \\
& \Phi_{\mathrm{a}}(\omega)=\frac{2 \mathrm{a}^{2}}{\Delta \mathrm{t}} \int_{0}^{\Delta t}(\Delta t-\tau) \cos \omega \tau d \tau
\end{aligned}
$$

Expandir.ng equation (7) yields

$$
\Phi_{\mathrm{a}}(\omega)=\frac{2 \mathrm{a}^{2}}{\Delta \mathrm{t}}\left[\int_{0}^{\Delta t} \Delta \mathrm{t} \cos \omega \tau \mathrm{d} \tau-\int_{0}^{\Delta t} \tau \cos \omega \tau \mathrm{d} \tau\right]
$$

Inteş̧rating equation (8) yields 
Hence, $\quad \Phi_{\mathrm{a}}(\omega)=\frac{2 \mathrm{a}^{2}}{\Delta \mathrm{t}}\left(\frac{1-\cos (\omega \Delta \mathrm{t})}{\omega^{2}}\right)$

$$
\Phi_{\mathrm{a}}(\omega)=\frac{2 \mathrm{a}^{2}}{\Delta \mathrm{t}}\left[\left.\frac{\Delta \mathrm{t}}{\omega} \sin \omega \tau\right|_{0} ^{\Delta t}-\left.\left(\frac{1}{\omega^{2}} \cos \omega \tau+\frac{\tau}{\omega} \sin \omega \tau\right)\right|_{0} ^{\Delta t}\right]
$$

But

$$
\begin{aligned}
& \sin ^{2}\left(\frac{\alpha}{2}\right)=\left(\frac{1-\cos \alpha}{2}\right), \text { let } \alpha=\omega \Delta t \text {, then equation (9) becomes } \\
& \Phi_{\mathrm{a}}(\omega)=\frac{4 \mathrm{a}^{2}}{\omega^{2} \Delta t} \sin ^{2}\left(\frac{\omega \Delta t}{2}\right) \\
& \Phi_{\mathrm{a}}(\omega)=\mathrm{a}^{2} \Delta \mathrm{t}\left(\frac{\sin (\omega \Delta \mathrm{t} / 2)}{\omega \Delta \mathrm{t} / 2}\right)^{2}
\end{aligned}
$$

Because pure white noise has an infinite bandwidth, the power is infinite. In real systems that receive white noise input, the noise bandwidth of the system limits the power. If the PSD function is flat and the frequency response of the system is flat, total noise power is calculated as the product of PSD and noise bandwidth, [2,5]. This is the case of physically realizable stationary white Gaussian noise process with band limited frequencies to $\pm \frac{1}{\pi \Delta t}$.

Using equation (10), the average power is calculated as follows

$$
\begin{aligned}
& P_{\text {avg }}=\phi_{a}(0)=\frac{1}{2 \pi} \int_{-\infty}^{\infty} \Phi_{a}(\omega) d \omega, \\
& P_{\text {avg }}=\frac{1}{2 \pi} \int_{-\infty}^{\infty} a^{2} \Delta t\left(\frac{\sin (\omega \Delta t / 2)}{\omega \Delta t / 2}\right)^{2} d \omega,
\end{aligned}
$$

Hence, $\quad P_{a v g}=a^{2}$.

By definition, the white noise has a constant power spectral density. It is impossible to obtain a noise that has a constant frequency spectrum from $-\infty$ to $+\infty$, because the signal would contain an infinite amount of power. However, it is possible to obtain a noise signal with flat frequency spectrum over a range of frequencies wider than the bandwidth of the system being analyzed.

The control system under study produces error measurements at discrete intervals of time, $\Delta$ t. The measurements are to be corrupted with white noise that has a constant PSD function $\Phi_{\mathrm{a}}^{\prime}$ the standard deviation of a set of samples taken $\Delta t$ seconds 
apart is then

$$
\sigma=\sqrt{\frac{\Phi_{\mathrm{a}}^{\prime}}{\Delta \mathrm{t}}}=\frac{\mathrm{a}}{\sqrt{\Delta \mathrm{t}}}
$$

Where: $\Phi_{\mathrm{a}}^{\prime}=\left\{\begin{array}{lr}a^{2}, & |\omega| \leq \frac{2}{\Delta t} \\ 0 & \text { elsewhere }\end{array}\right.$

The sample time $\Delta t$ represents time step of the numerical integration of the 6DOF simulation program. The problem is to determine the value of $\sigma$ for the random draws that are used to simulate noise digitally. The simulated white noise is shown in fig. (2).

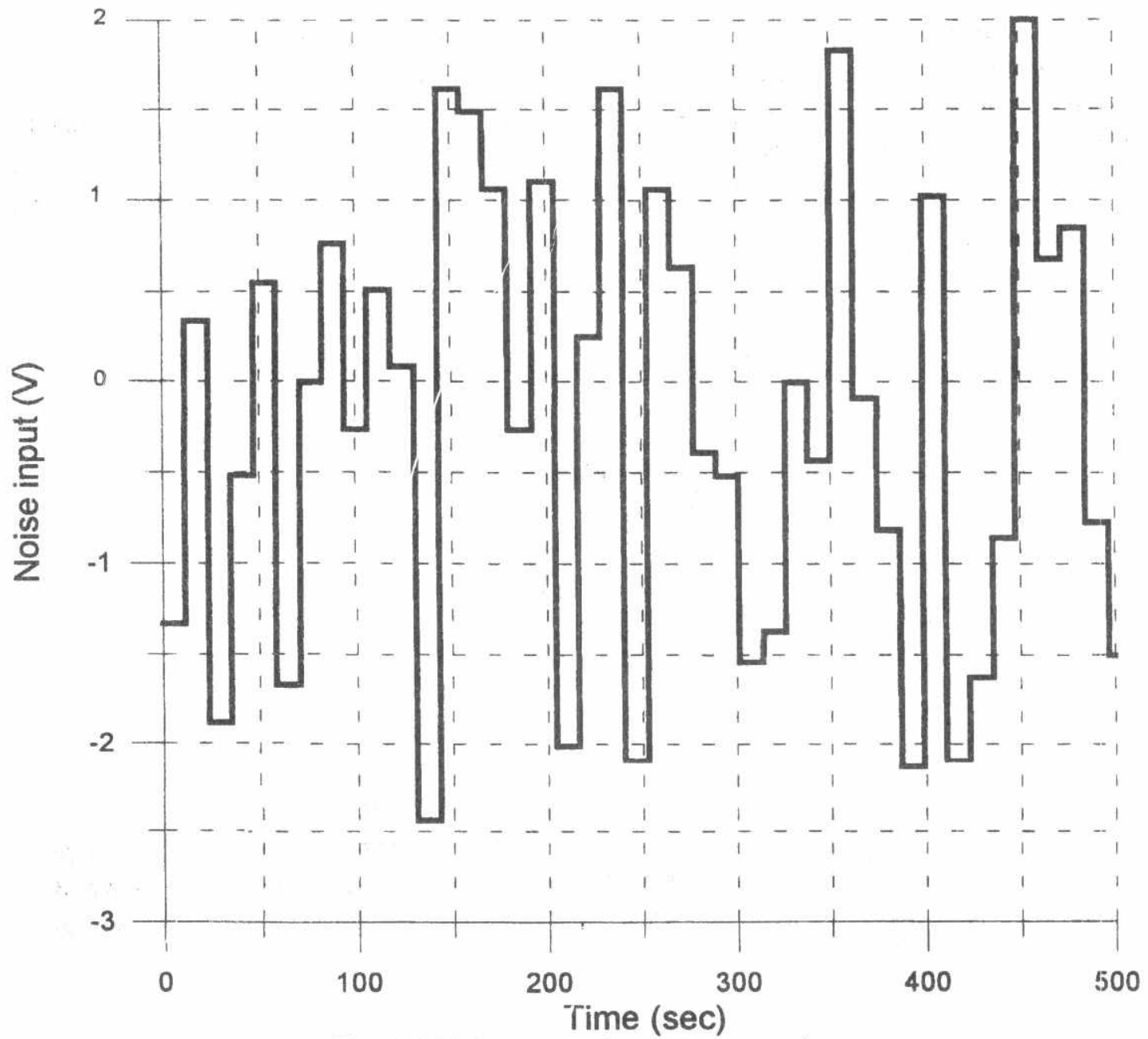

Fig. 2 White noise input versus time 


\section{GUIDANCE AND CONTROL LOOP OF THE HOMING GUIDED BOMB}

The guidance and control loop of the homing guided bomb given by reference [6] is shown in fig. 3. The input of the loop is the missile and target perturbations $Z_{B}$ and $Z_{T}$, respectively. $r$ is the range to go. The line-of-sight rate is sensed by the seeker or homing head which could be presented by a second order transfer function. The seeker parameters are seeker gain $K_{h}$, natural frequency $\omega_{n h}$, and damping ratio $\zeta_{h}$. The output of the seeker is the line-of-sight (LOS) rate $\dot{\lambda}_{p}$. This output is provided to the guidance module, which uses the proportional navigation guidance law to produce the desired acceleration command. $K_{P N}$ is the proportional navigation gain. The desired acceleration is the input to the autopilot loop.

The autopilot loop contains the fin servo that is considered as a gain $\mathrm{K}_{\mathrm{s}}$, the aerodynamic transfer functions $\left(J_{N} / \delta\right)$ and $(\dot{\theta} / \delta)$, and the accelerometer as a measurement device with gain $\mathrm{K}_{\mathrm{a}}$. The accelerometer is placed in front of $\mathrm{c} . \mathrm{g}$. in a distance $\mathrm{C}$ from nose. The actual normal acceleration is integrated twice to get the bomb displacement $Z_{\mathrm{B}}$. $\psi_{\text {fco }}$ is the flight path angle which is nearly equals zero. That is, the target is stationary or slow moving and so, the bomb need not to maneuver and will approach to the target in a nearly straight collision course. Hence, a very small flight path angle is expected; [7].

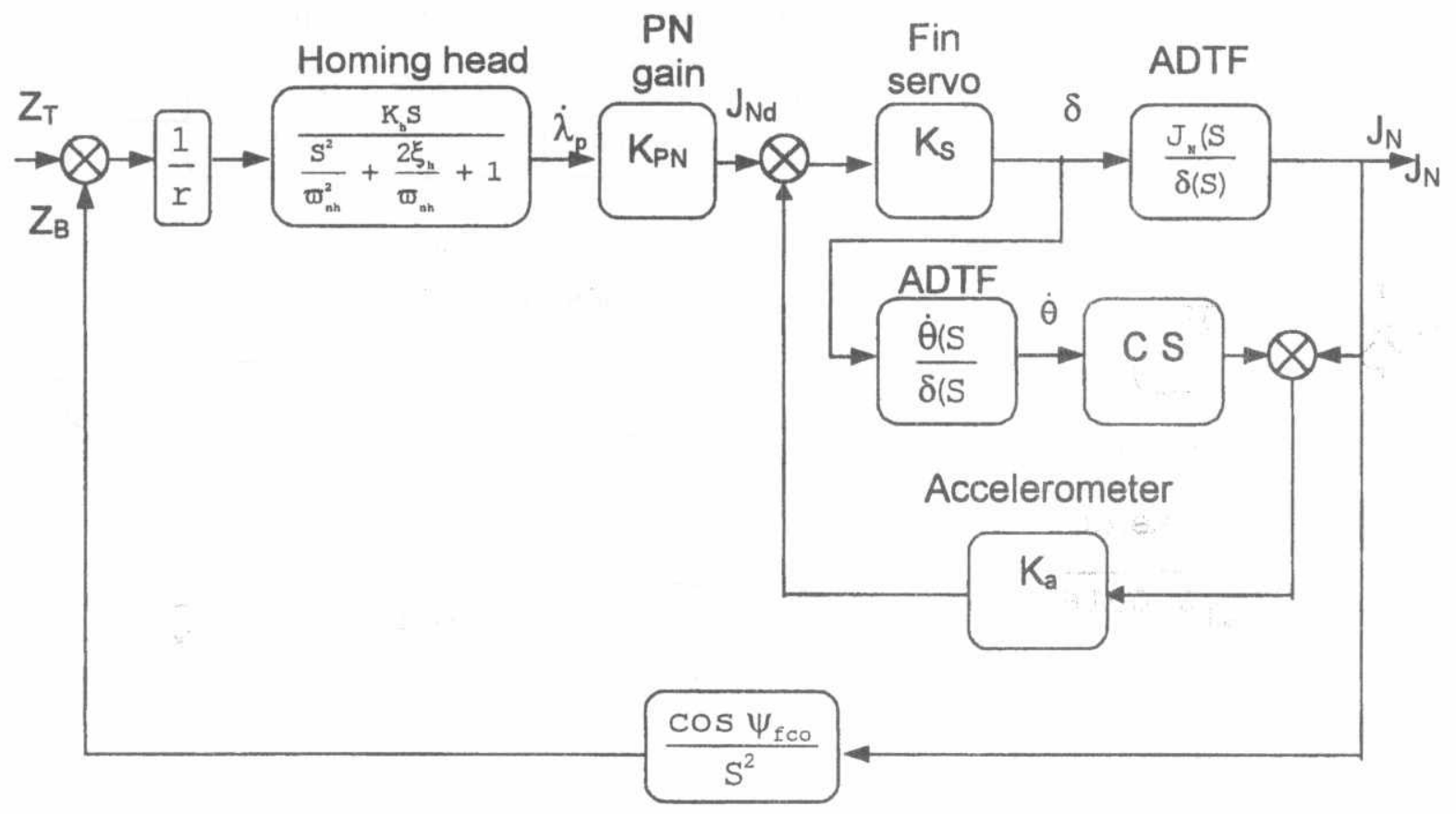

Fig. 3 Guidance and control loop of homing guided bomb

System evaluation represents one of the major tasks following the system design. The importance of system evaluation is further increased if the concerned 
system is a guidance system. The evaluation nature is extended from theoretical evaluation to realistic evaluation through practical experimentation and field tests. The developed 6-DOF simulation model represents the evaluation tool. The developed simulation computer program was run so many times and the flight results were recorded. Different releases of the bomb were considered for each run. The bomb release parameters include the bomb speed, position, and initial pitch angle.

For the ease of analysis, the motion of the bomb and target were considered in the vertical plane only. The targets are fixed or slowly moving targets such as tanks. During each run of the program, the various flight parameters of the bomb were recorded versus time from the launch instant till the impact time. The white noise is added to the desired input acceleration command and the flight parameters are compared for different signal-to-noise ratios.

The autopilot loop of the guided bomb under study has fin servo, aerodynamic transfer functions, and accelerometer. The aerodynamic transfer functions $\left(\mathrm{J}_{\mathrm{N}} / \delta\right)$ and $(\dot{\theta} / \delta)$ are calculated using linearization technique, [6]. The autopilot loop is shown in fig. (4).

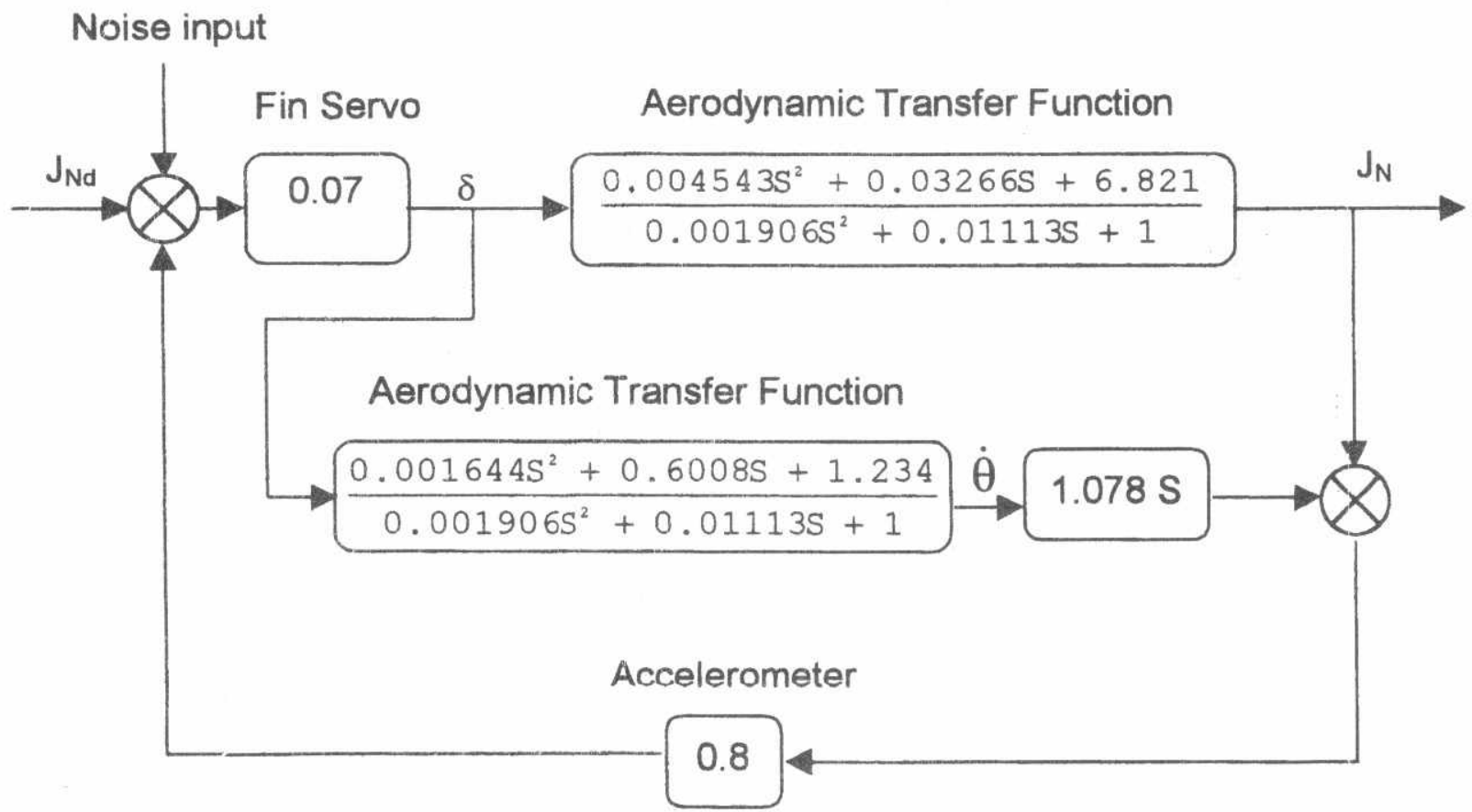

Fig. 4 Autopilot loop of homing guided bomb

The simulated noise has the response to the autopilot loop shown in fig. (5), [8]. Tr. 1e noise response is calculated for SNR equals $1 \mathrm{~dB}$. 


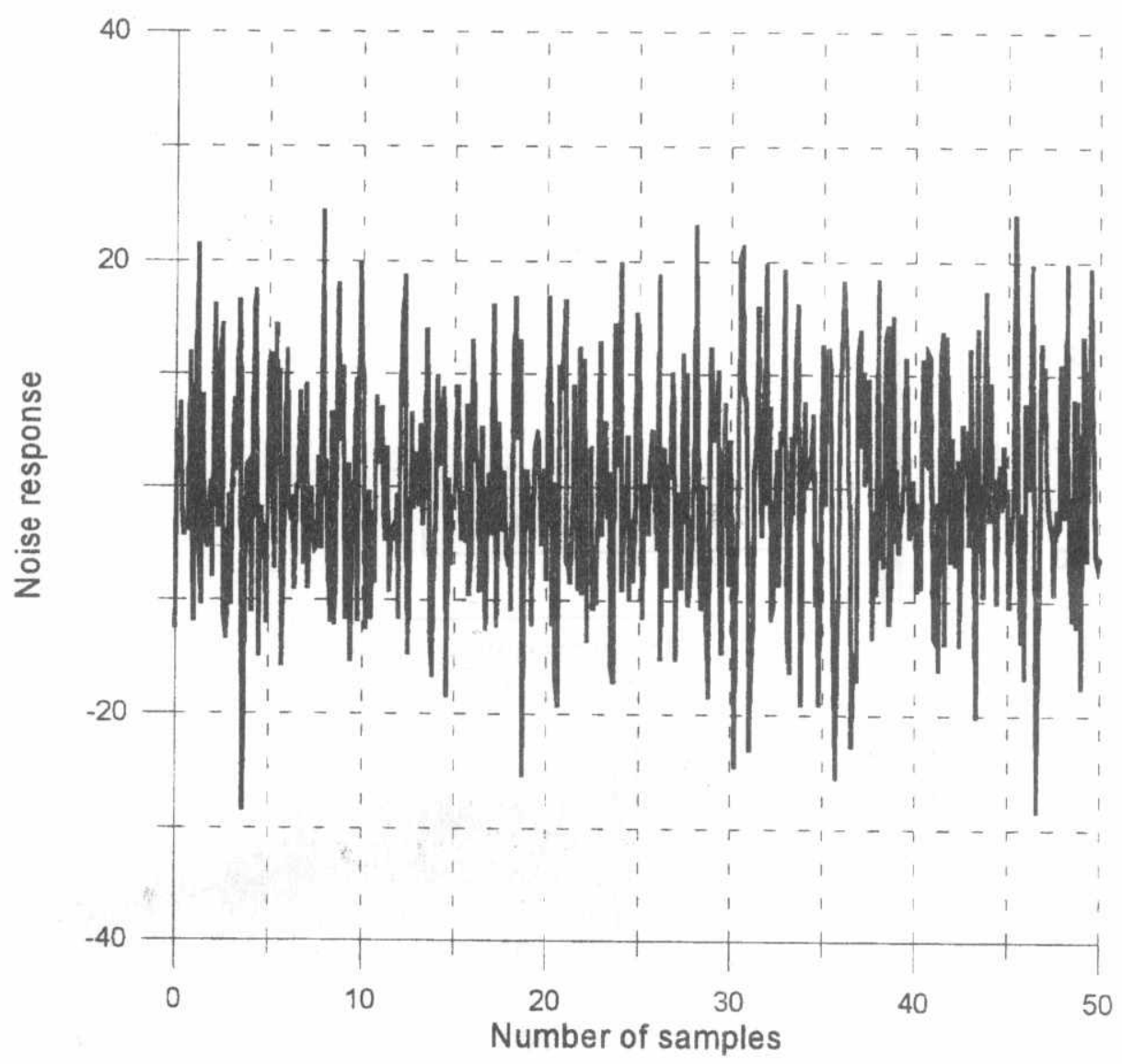

Fig. 5 Noise response of the autopilot loop $(S N R=1 \mathrm{~dB})$

The normal acceleration of the bomb with and without input noise is shown in fig. (6). The normal acceleration at the presence of noise has higher overshoot at the start time of guidance, which forces the bomb to exert more effort and high maneuver to follow the correct path. This is attributed to the fact that, if the bomb responds rabidly to a given command in the existence of noise, an excessive acceleration is imposed on the bomb airframe. For this value of SNR, equals $1 \mathrm{~dB}$, the bomb has missdistance equals 418 meters which is not a good performance. This will be shown in the trajectory curves for different SNR. 


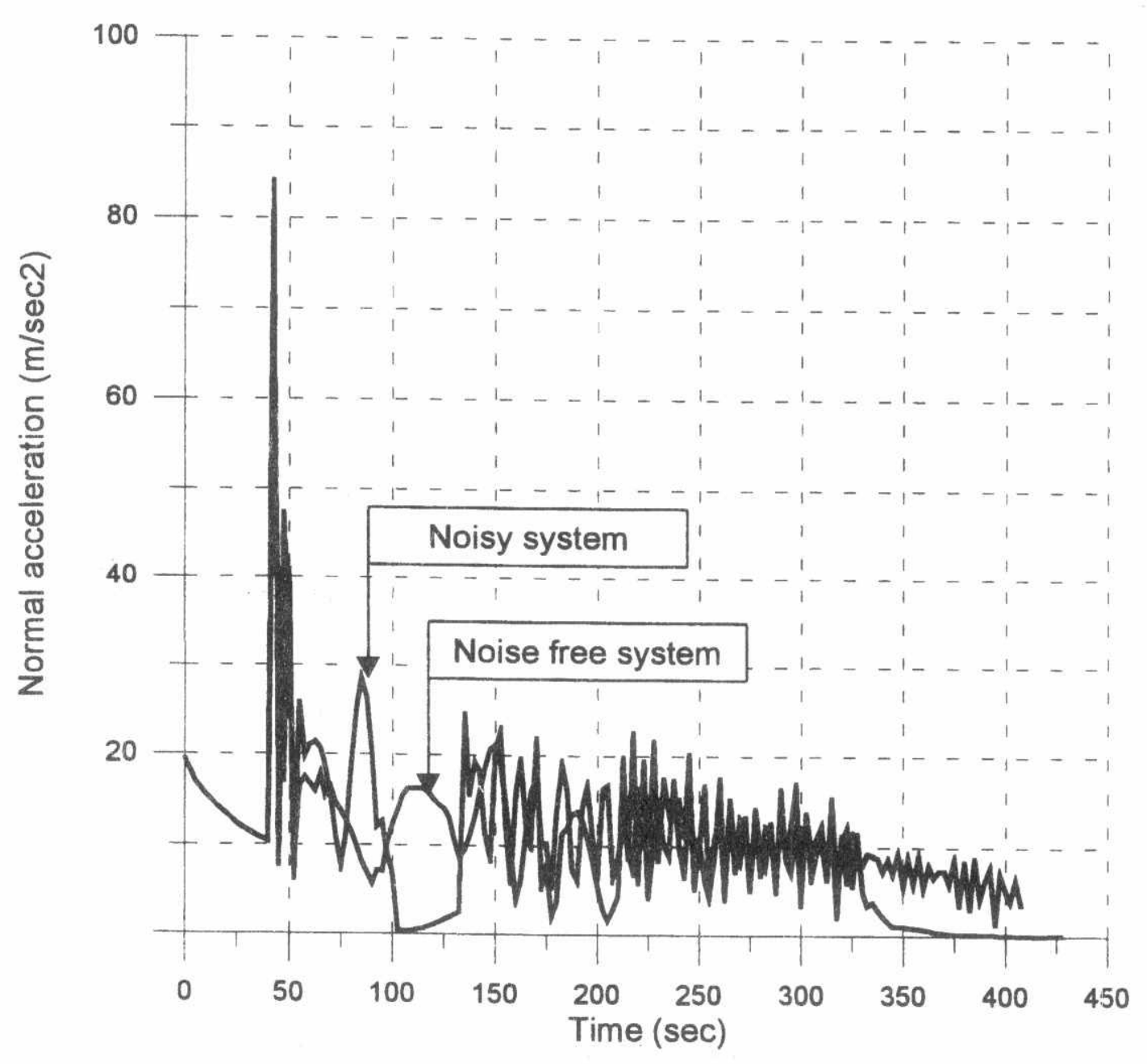

Fig. 6 Acceleration response of the autopilot loop without and with noise $(\mathrm{SNR}=1 \mathrm{~dB})$

Fig. (7) shows the trajectory in the vertical plane of a bomb launched with speed equals 2.5 Mach, at height equals $20 \mathrm{Km}$, and pitch angle equals $35^{\circ}$. The target is placed in the vertical plane with horizontal distance equals $60 \mathrm{Km}$ and is assumed a fixed target.

It is clear from fig. (7) that the bomb without noise input attacks the target with very small missdistance. The trajectory in the right represents the bomb without input noise. As SNR decreases, i.e. the noise signal increases, the bomb falls away from target, i.e. the missdistance increased. The bomb always falls before target and this is according to the fact that; when the noise level increases, the bomb moves away from the correct path and the control system produce suitable commands to nullify the existing error. So, the bomb exerts more effort and the allowable energy of the bomb is limited which makes it fall before the target position. 


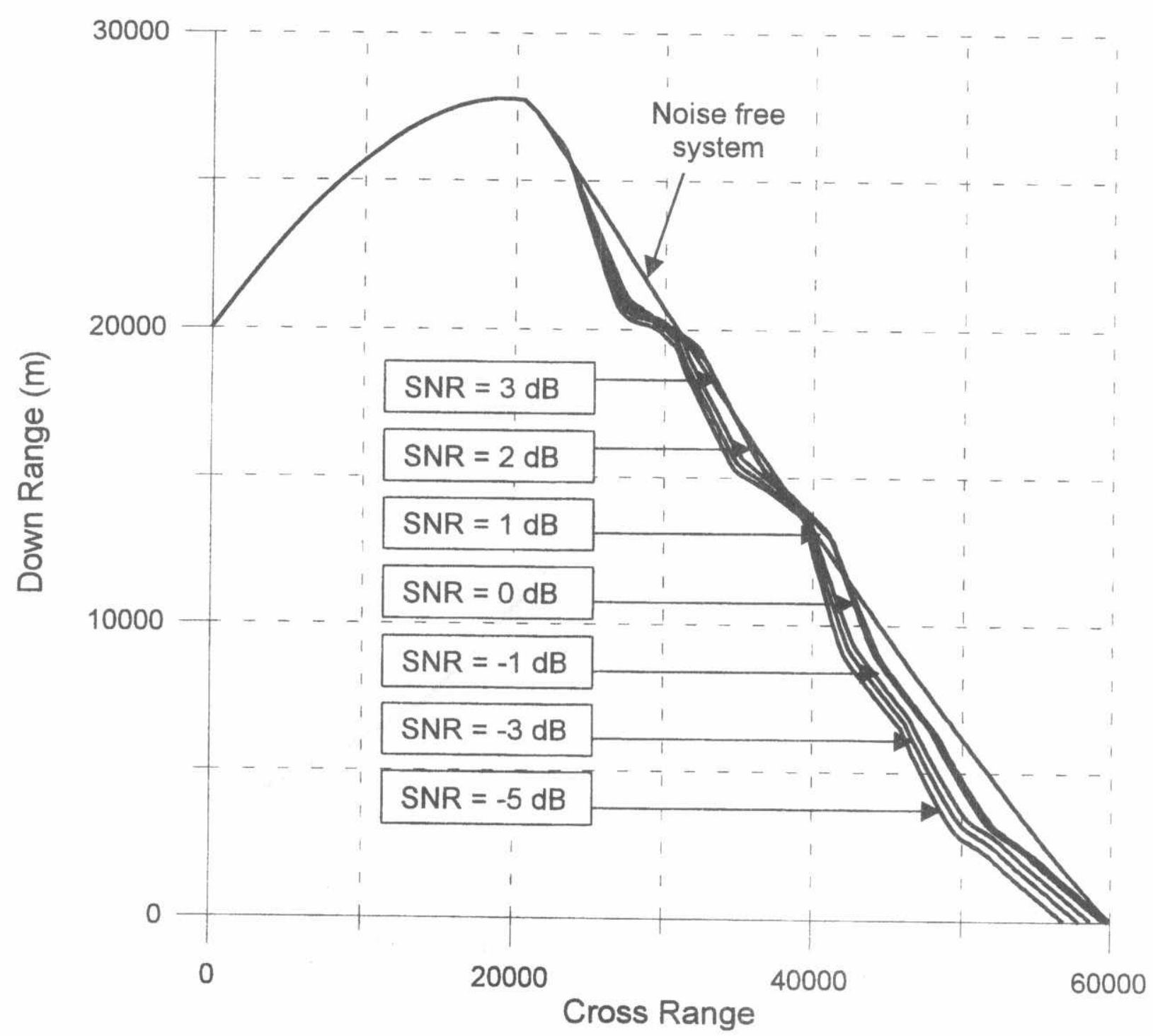

Fig. 7 Bomb Trajectory for different signal-to-noise ratio

The bomb-target missdistance versus SNR is shown in fig. (8). It is noted that the bomb falls before reaching the target position.

It is found from fig. (7) and (8) that the guided bomb will perform its mission correctly for SNR higher than or equal 3 . This value is chosen according suitable missdistance, equals 3.6 meters. If the SNR is higher than 3 , the missdistance will be smaller and tends to zero. The calculations were performed via the 6-DOF simulation program of the guided bomb. 


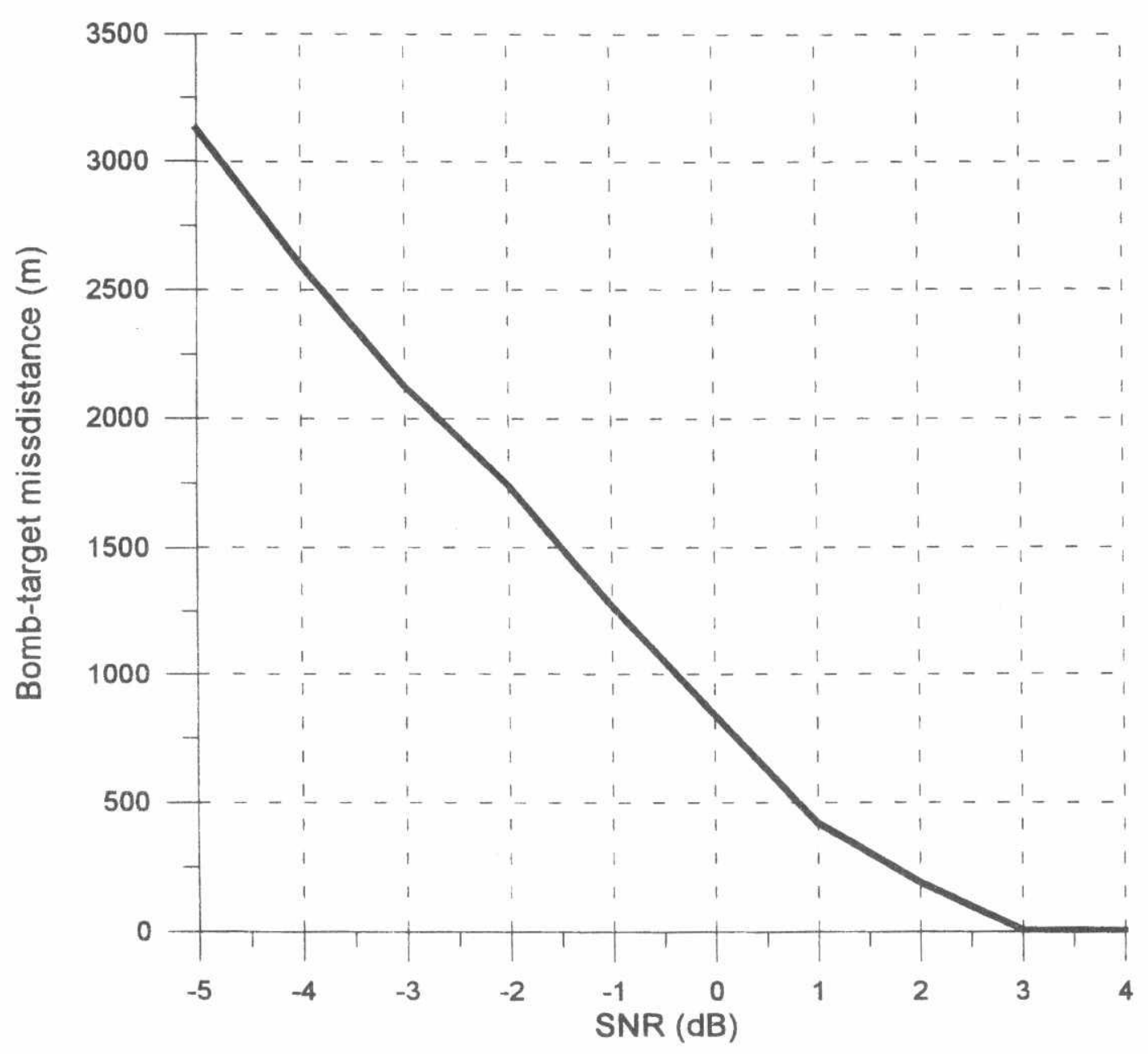

Fig. 8 Bomb-target missdistance for different signal-to-noise ratios

\section{Conclusions:}

Guidance and control loop evaluation and performance measurement represent important requirement following the loop design. There are many undesirable sources of disturbances that may affect the behavior of the bomb and erodes its performance during guidance phase.

The external simulated noise is considered as an atmospheric turbulence due to flight vibrations and oscillations coming from the environmental atmosphere. This noise is assumed to be zero-mean white Gaussian noise having flat frequency spectrum wider than the bandwidth of the analyzed system.

The guidance and control loop is analyzed throughout the normal acceleration, the bomb trajectory, and the final bomb-target missdistance. The noisy system has normal acceleration with large overshoot and wide variation that forces the bomb to 
exert more effort to overcome the noise effects and flies over the correct path to the target position. This effort consumes the bomb energy and causes it to fall before reaching the target. This is the reason of having large missdistance when applying high levels of noise to the system. This is clear from the trajectory and missdistance plot for different signal-to-noise ratios. Further investigation is required for the realization of compensators suitable to improve the guidance and control performance in the existence of noise.

\section{REFERENCES:}

[1] H.B. John, Automatic Control of Aircraft And Missiles, 2nd edition, Institute for Aerospace Studies, John Willey \& Sons, Inc., (1991).

[2] R. H. M. Macfadzean, Surface-Based Air Defense System Analysis, John Wiley \& Sons, Inc. ,(1995).

[3] R. C. Drof, R. H. Bishop, Modern Control Engineering, Addison-Wesley Publishing Company, Inc., (1995).

[4] Zarchan, Paul, Tactical and Strategic Missile Guidance, In Progress in Aeronautic and Astronautics, VOL. 124. Washington, DC, AIAA, (1990).

[5] E. Kreyszing, Advanced Engineering Mathematics, John Willey \& Sons, Inc., (1994).

[6] A. S. Gad, Msc, Design and experimentation for an autopilot for guided bombs, (1998).

[7] P. Garnell and D. J. East, Guided Weapon Control Systems, $2^{\text {nd }}$ edition, Pergamon press, New York,(1980).

[8] A. Grace, A.J. Laub, J.N. little, and C. Thompson, Control System Toolbox User's Guide, The MathWorks, Inc., 1990. 